\title{
Involvement of Human Amygdala and Orbitofrontal Cortex in Hunger-Enhanced Memory for Food Stimuli
}

\author{
J. S. Morris ${ }^{1,2,3}$ and R. J. Dolan ${ }^{1,4}$ \\ ${ }^{1}$ Wellcome Department of Cognitive Neurology, London WC1N 3BG, United Kingdom, 2Institute of Cognitive \\ Neuroscience, London WC1N 3AR, United Kingdom, 3/nstitute of Child Health, London WC1N 1EH, United Kingdom, \\ and ${ }^{4}$ Royal Free and University College Hospitals School of Medicine, London NW3 2DF, United Kingdom
}

We used positron emission tomography to measure regional cerebral blood flow (rCBF) in 10 healthy volunteers performing a recognition memory task with food and non-food items. The biological salience of the food stimuli was manipulated by requiring subjects to fast before the experiment and eat to satiation at fixed time points during scanning. All subjects showed enhanced recognition of food stimuli (relative to nonfood) in the fasting state. Satiation significantly reduced the memory advantage for food. Left amygdala rCBF covaried positively with recognition memory for food items, whereas rCBF in right anterior orbitofrontal cortex covaried with overall memory performance. Right posterior orbitofrontal rCBF covaried positively with hunger ratings during presentation of food items. Regression analysis of the neuroimaging data revealed that left amygdala and right lateral orbitofrontal rCBF covaried as a function of stimulus category (i.e., food vs non-food). These results indicate the involvement of amygdala and discrete regions of orbitofrontal cortex in the integration of perceptual (food), motivational (hunger), and cognitive (memory) processes in the human brain.

Key words: amygdala; orbitofrontal cortex; memory; food; hunger; satiety; functional neuroimaging
Cells in primate amygdala and orbitofrontal cortex respond to the sight, taste, and smell of food, as well as to stimuli associated with food reward (Sanghera et al., 1979; Nishijo et al., 1988; Rolls et al., 1990). Food-related neural activity in these regions is dependent, however, on the concurrent state of hunger or satiety: e.g., responses in amygdala and orbitofrontal cortex to food during fasting are suppressed after satiation (Rolls et al., 1989; Scott et al., 1995; Critchley and Rolls, 1996). These observations suggest that amygdala and orbitofrontal responses represent the biological or motivational significance of food stimuli and not simply the sensory properties of particular food items (LeDoux, 2000; Rolls, 2000). Other experiments have implicated the amygdaloid complex and orbitofrontal cortex in memory enhancement for emotionally arousing events, a process that appears to involve the release of systemic "stress" hormones, e.g., adrenaline and corticosterone (Cahill et al., 1996; Cahill and McGaugh, 1998; Hamann et al., 1999; Canli et al., 2000). However, although these motivational (i.e., hunger-satiety) and cognitive (i.e., mnemonic) phenomena have been studied separately in several experiments (Scott et al., 1995; Cahill et al., 1996), the interaction of these processes, particularly with respect to neural activity in amygdala and orbitofrontal cortex, has not been previously investigated.

In the present study, we used positron emission tomography (PET) to measure regional cerebral blood flow (rCBF) in healthy volunteers while they performed a recognition memory task involving food and non-food pictures. The motivational significance of the food stimuli was systematically manipulated by requiring each subject to perform the memory task in both fasting and sated

\footnotetext{
Received Feb. 9, 2001; revised April 23, 2001; accepted April 25, 2001.

This work was supported by grants from the Wellcome Trust to J.S.M. and R.J.D. Correspondence should be addressed to Prof. Dolan, Wellcome Department of Cognitive Neurology, 12 Queen Square, London WC1N 3BG, UK. E-mail: r.dolan@fil.ion.ucl.ac.uk.

Copyright (C) 2001 Society for Neuroscience $0270-6474 / 01 / 215304-07 \$ 15.00 / 0$
}

states. We conjectured that food items would be better remembered than non-food in the fasting state and that satiation would reduce this memory advantage. In light of previous data, we predicted that hunger-related modulation of memory for food items would be reflected in amygdala and orbitofrontal cortex activations (Rolls et al., 1989; Scott et al., 1995; Critchley and Rolls, 1996), and, moreover, that task-dependent interactions between responses in these regions would be observed (Schoenbaum et al., 1998; Baxter et al., 2000). On the basis of previous neuroimaging studies (Tataranni et al., 1999; Liu et al., 2000), we also predicted that neural activity in hypothalamus and insula would covary primarily with physiological state (i.e., hungersatiety) rather than with stimulus category (food-non-food) or cognitive (memory) performance.

\section{MATERIALS AND METHODS}

Subjects. Ten right-handed volunteer subjects were recruited by advertisement. None of the subjects had any past history of neurological or psychiatric disorder (including eating disorders). All subjects were medication-free at the time of experiment. Table 1 gives details of each subject's sex, age and body mass index. All subjects gave informed consent to the study, which was approved by the Ethics Committee of the National Hospital for Neurology and Neurosurgery and the United Kingdom Administration of Radioactive Substances Advisory Committee.

Experimental design. Subjects were instructed to abstain from eating in the $16 \mathrm{hr}$ preceding the scanning session (which always began at 4:00 P.M.) but to drink fluids as normal. All subjects had 12 PET scans at regular $8 \mathrm{~min}$ intervals. Each scan was $90 \mathrm{sec}$ in duration. At fixed time points between individual scans, subjects were asked to eat to satiation while remaining in the scanner. They were provided with a large cheese and salad sandwich and two sweets (slices of apple and chocolate cakes) followed by a drink of water. Subjects were randomized into two groups: (1) an "early satiation" group who ate after four PET scans (i.e., $\sim 24 \mathrm{~min}$ from beginning of scanning, and (2) a "late satiation" group who ate after eight PET scans (i.e., $\sim 56 \mathrm{~min}$ from beginning of scanning). The interval between the fourth and fifth scans in the early group and between the eighth and ninth scans in the late group was $\sim 20 \mathrm{~min}$. Immediately 
Table 1. Ages and body mass indices of subjects

\begin{tabular}{llll} 
Subject & Sex & $\begin{array}{l}\text { Age } \\
(\text { years })\end{array}$ & $\begin{array}{l}\text { Body mass index } \\
\left(\mathrm{kg} / \mathrm{m}^{2}\right)\end{array}$ \\
\hline 1 & M & 19.8 & 19.88 \\
2 & M & 21.0 & 19.97 \\
3 & M & 31.2 & 25.10 \\
4 & F & 59.5 & 32.77 \\
5 & M & 24.5 & 25.83 \\
6 & M & 24.1 & 23.15 \\
7 & M & 31.2 & 23.94 \\
8 & M & 44.6 & 24.49 \\
9 & M & 22.0 & 23.20 \\
10 & M & 22.6 & 21.13 \\
\hline Mean & & 30.05 & 23.95
\end{tabular}

before each PET scan, subjects rated their feelings of hunger and satiety on a $0-100$ scale $(0$, most hungry; 100 , most sated that subjects could ever imagine themselves). These subjective scores were used to create a hunger rating covariate, such that 0 on the subjective rating scale (i.e., maximum hunger score) was assigned a value of 100 and 100 on the subjective scale (i.e., maximum satiety score) assigned a value of 0 . Before the first scan, and after the last scan, blood samples were taken from every subject to measure plasma levels of glucose, insulin, hydroxybutyrate, and free fatty acids. The body mass index (weight in kilograms/ (height in meters) ${ }^{2}$ ) was also determined for each subject.

Five minutes before each PET scan, subjects were instructed to commit to memory 10 pictures, each shown singly for $4 \mathrm{sec}$ on a computer monitor screen. Two categories of pictures were shown: (1) food, consisting of color photographs of a range of appetizing food, both sweet and savory, taken from cookbooks; and (2) non-food, consisting of color photographs of household objects (chairs, sofas, lamps, mirrors, tools, ornaments, etc.) that had no association with eating (i.e., no crockery, cutlery, kitchen utensils, etc.). All 10 pictures in the same sequence belonged to the same category. During the following PET scan, these 10

A

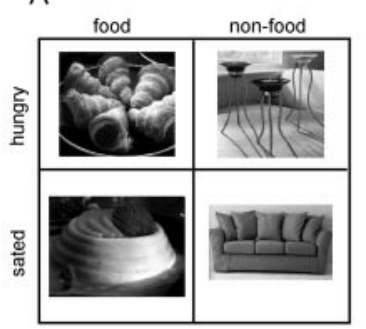

B
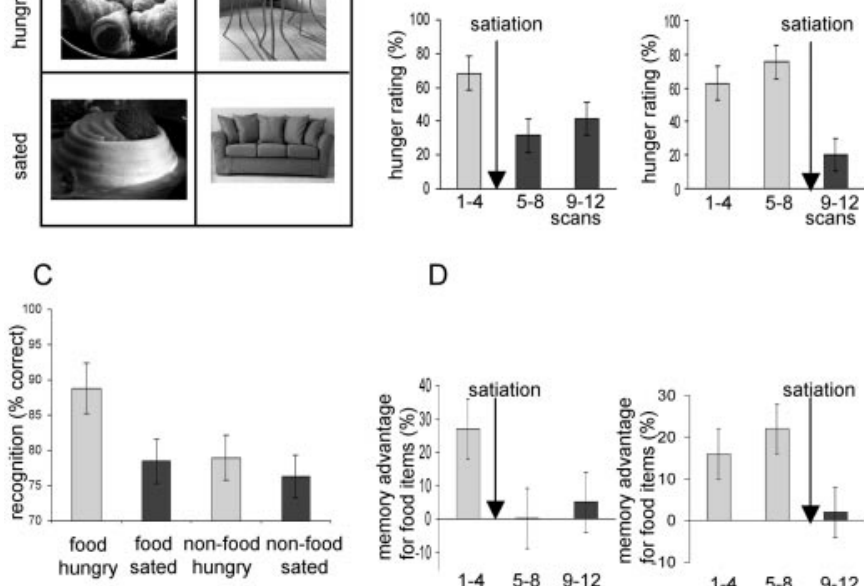

D

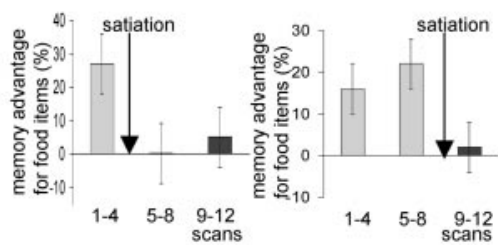

Figure 1. A, Two $\times$ two factorial experimental design. Food and nonfood stimuli were presented in both hungry and sated states. $B$, Mean hunger ratings for early and late satiation groups displayed across three scanning blocks (i.e., scans $1-4,5-8$, and 9-12). $C$, Mean recognition memory scores in all four conditions. $D$, Mean memory advantage for food items over non-food in early and late satiation groups. Memory advantage was calculated by subtracting non-food recognition scores from food recognition scores in each of the three scanning blocks. In $B-D$, bars represent 2 SEs, and arrows indicate time of satiation.
Table 2. Levels of plasma glucose and insulin

\begin{tabular}{|c|c|c|c|c|}
\hline \multirow[b]{2}{*}{ Subject } & \multicolumn{2}{|c|}{$\begin{array}{l}\text { Glucose }(\mathrm{mmol} / \mathrm{l}) \\
\text { reference range: } \\
3.3-9.0\end{array}$} & \multicolumn{2}{|c|}{$\begin{array}{l}\text { Insulin }(\mathrm{pmol} / \mathrm{l}) \\
\text { reference range: } \\
<17\end{array}$} \\
\hline & Fasting & Sated & Fasting & Sated \\
\hline 1 & 3.7 & 6.8 & 2.7 & $42.7^{b}$ \\
\hline 2 & 4.9 & 6 & 9.1 & $32.5^{b}$ \\
\hline 3 & 4.7 & 6.3 & $a$ & $a$ \\
\hline 4 & 4.3 & 5.1 & $a$ & $a$ \\
\hline 5 & 3.9 & 5.4 & 8.2 & $43.0^{b}$ \\
\hline 6 & 4.2 & 5.6 & 4.1 & $76.0^{b}$ \\
\hline 7 & 4.5 & 5.9 & 8.8 & $73.7^{b}$ \\
\hline 8 & 4.4 & 7 & 2.5 & $24.2^{b}$ \\
\hline 9 & 3.7 & 5.4 & 1.9 & $29.9^{b}$ \\
\hline 10 & 4.2 & 6.6 & 3.8 & $32.5^{b}$ \\
\hline Mean & 4.3 & 6.0 & 5.1 & $44.3^{b}$ \\
\hline
\end{tabular}

${ }^{a}$ Measurements lost because of technical problems; ${ }^{b}$ outside reference range.

pictures were shown again in a random sequence with 10 new pictures of the same category. All pictures were shown singly for $4 \mathrm{sec}$. With each picture presentation, subjects were instructed to indicate via right-hand button presses whether they had or had not seen the picture $5 \mathrm{~min}$ before. Subjects made responses on every trial, pressing one button for "yes" (old) and another button for "no" (new). Each subject had six food scans and six non-food (household object) scans. The order of food and non-food conditions was counterbalanced within and across subjects. The early satiation subject group had two food and two house scans before eating; the late group had four food and four house scans before satiation. Subjects were given a score for each of their responses to the 20 pictures in the scanning (test) sequence: i.e., +1 for correct identification either of an "old" (repeated) picture or of a "new" picture, and 0 for an incorrect or absent response, making a total maximum score of 20 $(100 \%)$.

Neuroimaging. Subjects had 12 scans of the distribution of $\mathrm{H}_{2}{ }^{15} \mathrm{O}$ acquired with a Siemens/CTI ECAT EXACT HR ${ }^{+}$PET scanner operated in high-sensitivity three-dimensional mode. Subjects received a total of $350 \mathrm{MBq}$ of $\mathrm{H}_{2}{ }^{15} \mathrm{O}$ intravenously over $20 \mathrm{sec}$. A Hanning filter was used to reconstruct the images into 63 planes, resulting in a $6.4 \mathrm{~mm}$ transaxial and $5.7 \mathrm{~mm}$ axial resolution (full width half maximum).

Spatial preprocessing. The PET scans were initially realigned using sinc interpolation to remove movement artifacts before being transformed into a standard stereotactic space. Structural MRIs from each subject were co-registered into the same space. A Gaussian filter set at $12 \mathrm{~mm}$ full width at half maximum was used to smooth the PET data, which

\section{Table 3. Levels of plasma hydroxybutyrate and free fatty acids ${ }^{a}$}

\begin{tabular}{|c|c|c|c|c|}
\hline \multirow[b]{2}{*}{ Subject } & \multicolumn{2}{|c|}{$\begin{array}{l}\text { Hydroxybutyrate } \\
\text { reference range: } \\
50-200 \mathrm{~mol} / 1\end{array}$} & \multicolumn{2}{|c|}{$\begin{array}{l}\text { Free fatty acids } \\
\text { reference range: } \\
0.1-0.6 \mathrm{~mol} / 1\end{array}$} \\
\hline & Fasting & Sated & Fasting & Sated \\
\hline 1 & $863^{b}$ & 172 & $1.11^{b}$ & $0.61^{b}$ \\
\hline 2 & 138 & $31^{b}$ & $0.66^{b}$ & $0.77^{b}$ \\
\hline 3 & 82 & $42^{b}$ & $1.04^{b}$ & 0.44 \\
\hline 4 & $a$ & $a$ & $a$ & $a$ \\
\hline 5 & 110 & 75 & $0.73^{b}$ & 0.48 \\
\hline 6 & $264^{b}$ & 104 & $1.05^{b}$ & 0.24 \\
\hline 7 & $325^{b}$ & 185 & $0.93^{b}$ & $0.68^{b}$ \\
\hline 8 & $359^{b}$ & $37^{b}$ & $1.3^{b}$ & 0.14 \\
\hline 9 & $370^{b}$ & 55 & $0.83^{b}$ & 0.12 \\
\hline 10 & 65 & $21^{b}$ & $1.99^{b}$ & 0.10 \\
\hline Mean & $286^{b}$ & 80 & $1.07^{b}$ & 0.4 \\
\hline
\end{tabular}

${ }^{a}$ Measurements lost because of technical problems; ${ }^{b}$ outside reference range. 
A

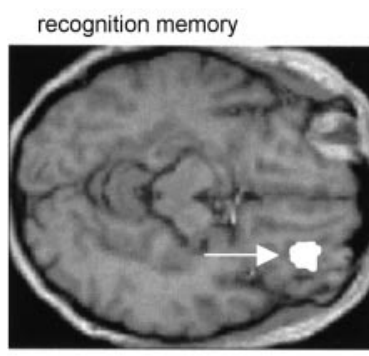

right orbitofrontal cortex $30,42,-16$

C

food-memory interaction

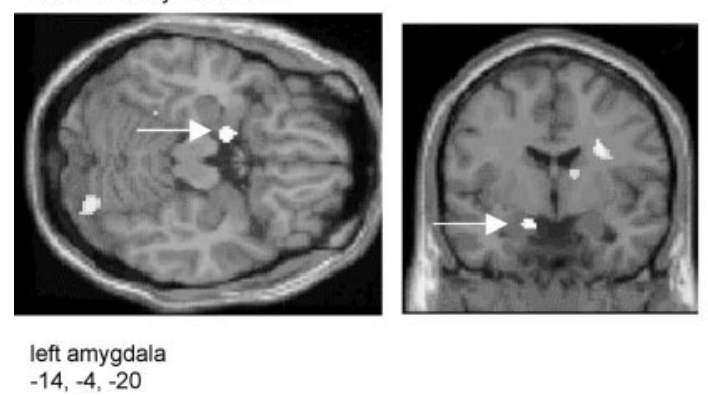

B
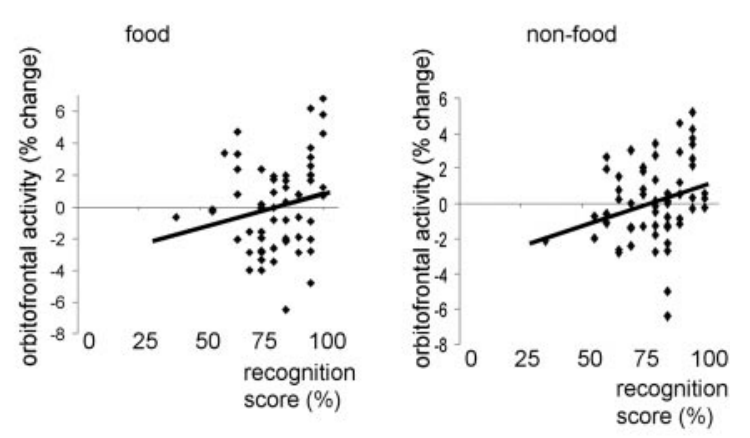

D
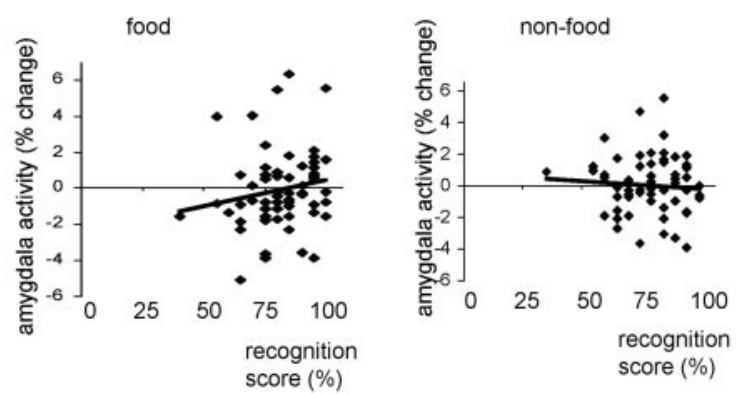

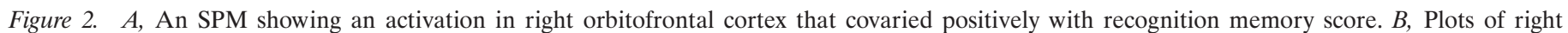

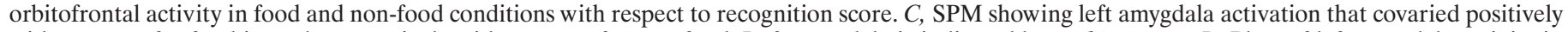

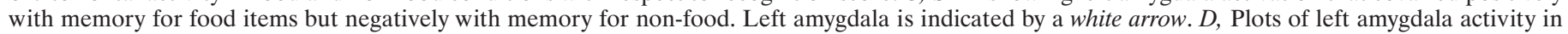

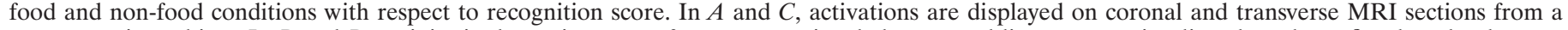

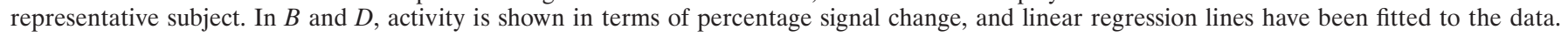

were adjusted to a global mean of $50 \mathrm{ml} \cdot \mathrm{dl}^{-1} \cdot \min ^{-1}$. SPM99 was used for all spatial preprocessing (Friston et al., 1995).

Statistical analysis. Subjects' ratings of hunger before each scan and their memory recognition scores were used as subject-specific covariates of interest in a statistical analysis of the PET data using SPM99. Food and non-food conditions were specified for both fasted and sated states (Fig. 1A). The chronological order of scans was specified as a confounding covariate. The data were globally normalized using a subject-specific ANCOVA. Specific effects were tested by applying linear contrasts to the parameter estimates for the variables of interest. The resulting $t$ statistic at every voxel constitutes a statistical parametric map (SPM). Contrasts were specified for individual subjects, and group effects were assessed by conjunction analyses of subject-specific contrasts. Reported $p$ values for $a$ priori regions of interest (i.e., amygdala, insula, hypothalamus, and orbitofrontal cortex) are corrected for the number of comparisons made within each region (Worsley et al., 1996). Anatomical localization of all activations was confirmed by coregistration with individual subjects' MRIs.

Functional data from a left amygdala voxel $(x=-12, y=-6, z=$ -18 ), maximally activated in the contrast of memory for food versus memory for non-food, were entered into a separate regression analysis to test for psychophysiological interactions (Friston et al., 1997). Brain regions were identified where covariation with left amygdala rCBF changed as a function of stimulus category (i.e., food vs non-food).

\section{RESULTS}

\section{Biochemical data}

All subjects maintained their plasma glucose within the normal range during both fasted and sated states and showed appropriate increases in plasma insulin after food ingestion (Table 2). Levels of free fatty acids in the fasting state were significantly elevated beyond the normal range in all subjects (Table 3 ). Mean hydroxybutyrate levels were also significantly elevated during fasting (Table 3).

\section{Behavioral data}

All 10 subjects showed a similar pattern in subjective ratings of hunger (Fig. $1 B$ ). Hunger ratings were initially moderately high (fasting mean, 68.8 points; SD, 11.4 points), increased gradually until satiation, and then fell abruptly (post-satiation mean, 31.1points; SD, 12.3). Mean fasting and post-satiation hunger ratings were significantly different when tested with a one-tailed two-sample $t$ test $(t=17.4 ; p<0.001)$. After the steep satiationrelated decrease, hunger ratings tended to increase slowly during the remainder of the session (Fig. $1 B$ ).

Enhanced recognition of food stimuli in the fasting state was seen in all subjects (Fig. 1C). Food items showed increased recognition scores compared with non-food in the pre-satiation period $(t=3.95 ; p<0.001)$. After satiation, however, there was no significant difference in recognition score between food and non-food items $(t=0.95 ; p>0.1)$. The interaction between physiological state (i.e., hunger-satiety) and stimulus category (i.e., food-non-food) was significant $(t=3.42 ; p<0.001)$. It is important to note that memory performance scores included "rejections" (i.e., successfully identifying new pictures) as well as "hits" (identifying old items). Changes in memory performance cannot be attributable, therefore, simply to a change in response bias, e.g., subjects tending to respond "old" more often in the fasting state. Moreover, the category-specific improvement in recognition scores was closely related to subjects' concurrent state of hunger (Fig. 1B,D). In the early satiation subject group, memory advantage for food was abolished in post-eating scans $5-8$, in line with decreased hunger ratings (Fig. 1D). In the late satiation group, memory advantage for food increased in scans 5-8, in line 
with increased hunger ratings and was abolished in post-satiation scans 9-12 (Fig. 1D).

\section{Neuroimaging data}

Right anterior orbitofrontal cortex activity $(x=30, y=42, z=$ -16 ; $p<0.05$, corrected) covaried positively with overall recognition memory score (i.e., memory for both food and non-food stimuli) (Fig. $2 A, B)$. By contrast, activity in left amygdala $(x=$ $-14, y=-4, z=-20 ; p<0.01$, corrected) covaried positively with memory for food stimuli, but negatively with memory for non-food items (Fig. 2C,D). A left dorsal insula region $(x=-42$, $y=2, z=-2 ; p<0.05$, corrected) showed a similar categoryspecific pattern of response covariation to that observed in left amygdala. Other regions positively covarying with overall memory score included bilateral cerebellum $(x=-4, y=-68, z=$ -46 and $x=24, y=-56, z=-50 ; p<0.001$, uncorrected) and bilateral parietal cortex $(x=-28, y=-52, z=44$ and $x=48, y=$ $-46, z=-56$; $p<0.001$, uncorrected). However, activations in these areas, which were not a priori regions of interest, did not reach a corrected level of significance.

In a separate regression analysis, we used measures of activity from the maximally activated voxel $(x=-14, y=-4, z=-20)$ in left amygdala to investigate how this structure functionally interacts with other brain regions. We first determined how left amygdala $\mathrm{rCBF}$ covaried with activity in every other brain voxel during both food and non-food scans. Then, to test for psychophysiological interactions (Friston et al., 1997), we contrasted, at every brain voxel, the slopes of the regression lines associated with these different stimulus-dependent rCBF covariations (i.e., food vs non-food). This analysis showed that $\mathrm{rCBF}$ in right lateral orbitofrontal cortex $(x=38, y=30, z=-24 ; p<0.01$, corrected $)$ covaried positively with left amygdala activity during food scans, but negatively during non-food scans (Fig. 3). This orbitofrontal area (Fig. $3 A$ ) was adjacent $(8 \mathrm{~mm}$ lateral and $12 \mathrm{~mm}$ posterior) to the orbitofrontal region associated with overall memory recognition (Fig. 2A).

Activity in a region to the right of the midline, encompassing both hypothalamus $(x=-6, y=2, z=-8$; $p<0.05$, corrected) and nucleus accumbens $(x=-12, y=10, z=0 ; p<0.001$, uncorrected) covaried positively with overall ratings of hunger (Fig. 4A,B). Activity in this region was clearly "time-locked" to the period of satiation, falling to its lowest level in scans 5-8 in the early satiation group, but rising to its highest level in scans 5-8 in the late satiation group (Fig. 4B). The close parallel between these contrasting response patterns and group-specific changes in hunger rating (Fig. $1 B$ ) exclude the possibility that this effect is attributable simply to time-dependent factors. Activity in right anterior insula ( $x=34, y=24, z=-8 ; p<0.001$, corrected) also covaried positively with hunger ratings, with a similar time-locked pattern of response across all subjects (Fig. 4C,D). Interestingly, a separate region of right insula $(x=42, y=-2, z=-6 ; p<0.05$, corrected), $26 \mathrm{~mm}$ posterior to the "hunger-related" region, covaried with satiety, i.e., covaried negatively with hunger ratings (Fig. 5). Comparison of the $\mathrm{rCBF}$ in this posterior insula region in the early and late satiation groups again revealed a close relationship to time of satiation (Fig. $5 B$ ).

Right posterior orbitofrontal cortex $\operatorname{rCBF}(x=34, y=12, z=$ $-24 ; p=0.065$, corrected; $p<0.001$, uncorrected) showed a category-dependent covariation with hunger ratings (Fig. 6). Orbitofrontal $\mathrm{rCBF}$ covaried positively with hunger in the food condition but showed no significant covariation with hunger in the non-food condition (Fig. 6B). This food-hunger-related region of
A

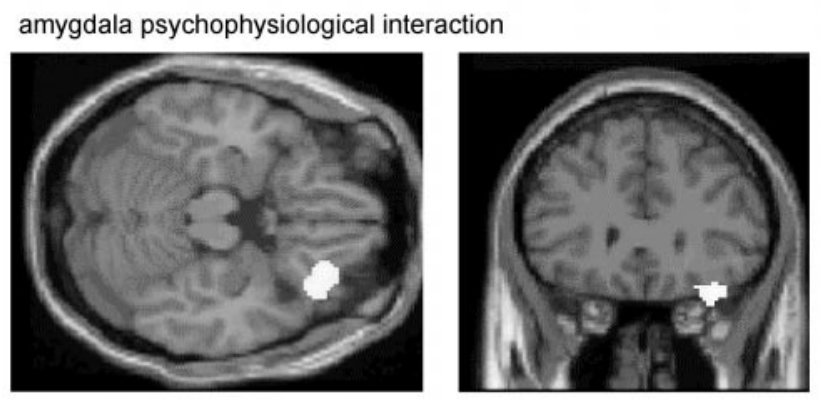

right orbitofrontal cortex

$38,30,-24$

B
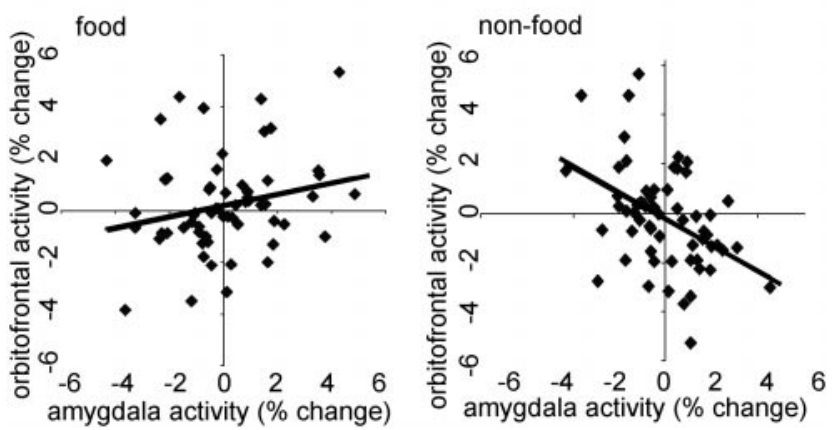

Figure 3. A, An SPM showing a psychophysiological interaction between right orbitofrontal cortex and left amygdala rCBF. Measures of rCBF in maximal amygdala voxel $(x=-14, y=-4, z=-20)$ were used as a covariate of interest in a condition-dependent regression analysis. The orbitofrontal activation is displayed on coronal and transverse MRI sections from a representative subject. $B$, Plots of right orbitofrontal activity in food and non-food conditions with respect to left amygdala activity. Activations are shown in terms of percentage signal change, and a linear regression line has been fitted to the data.

orbitofrontal cortex (Fig. 6) was located $30 \mathrm{~mm}$ posterior to the memory-related area (Fig. 2) and $18 \mathrm{~mm}$ posterior to the amygdala-related region (Fig. 3). It is noteworthy that mean $\mathrm{rCBF}$ in right posterior orbitofrontal cortex was not significantly different between food and non-food conditions (Fig. 6B). Indeed, no brain region exhibited significantly increased (or decreased) mean $\mathrm{rCBF}$ to food items (relative to non-food) in either fasting or sated states. However, inspection of individual subject data revealed that 3 of the 10 subjects had significant increases in left amygdala $\mathrm{rCBF}$ to food items in the fasting state, whereas the other 7 subjects had increased left amygdala rCBF to food in the sated state. These striking individual differences in food-evoked amygdala $\mathrm{rCBF}$ were not related to any physical, biochemical, or behavioral variable measured in the present study.

\section{DISCUSSION}

In this study, we demonstrate a category-specific (food-related) interaction between hunger (motivational state) and cognitive performance (recognition memory). A close, time-locked relationship between hunger ratings and memory for food was evident in both early and late satiation groups (Fig. 1B,D). Most notably, in the $20 \mathrm{~min}$ interval between pre-satiation and postsatiation scans, a $>20 \%$ memory advantage for food stimuli relative to non-food was abolished in all 10 subjects (Fig. 1C,D). 
A

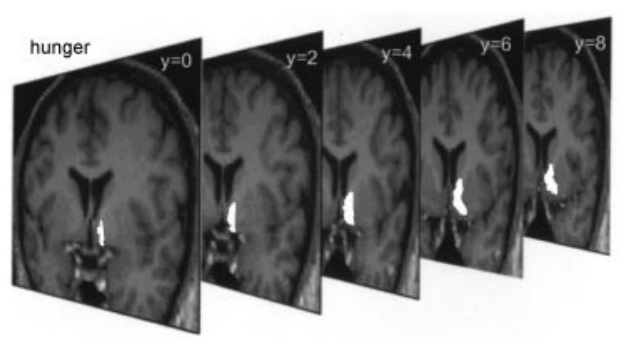

Figure 4. A, An SPM showing activations in hypothalamus and ventral striatum (nucleus accumbens) that covaried positively with subjective ratings of hunger. The activations are displayed on serial coronal MRI sections from a representative subject. $B$, Mean hypothalamic activity across three scanning blocks (i.e., scans 1-4, 5-8, and 9-12) are shown for early and late satiation groups. $C$, An SPM showing an activation in right insula that covaried positively with subjective ratings of hunger. The activation is displayed on sagittal and transverse MRI sections from a representative subject. Right insula is indicated by a white arrow. D, Mean insula activity is shown (in terms of percentage signal change) for early and late satiation groups. In $B$ and $D$, bars represent 2 SEs, and arrows indicate time of satiation.
C

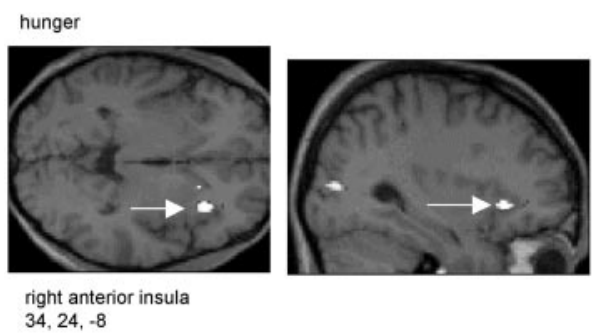

$$
\begin{array}{ll}
\text { hypothalamus } & \text { nucleus accu } \\
6,2,-8 & 12,10,0
\end{array}
$$

B

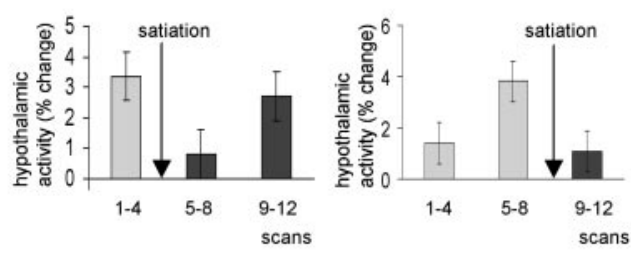

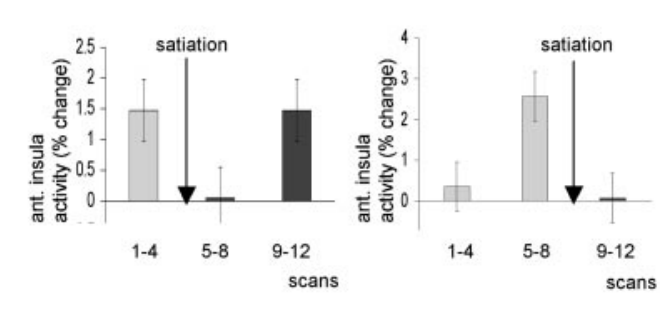

Previous psychophysical experiments have shown that recognition of tachistoscopically presented food-relevant words is enhanced during fasting compared with satiation (Erwin and Ferguson, 1979; Ferguson, 1983). Our behavioral data show a similar interaction between physiological, motivational, and cognitive processes, thus uniting emotional memory enhancement (Cahill and McGaugh, 1998) and motivational modulation of behavioral preference (Rolls et al., 1982) within a single experimental paradigm.

Amygdala and orbitofrontal cortex rCBF were both associated with recognition memory performance (Fig. 2). Left amygdala $\mathrm{rCBF}$ covaried specifically with memory for food stimuli (Fig. $2 C, D)$. This result accords with previous neuroimaging data showing that recognition memory of emotional (compared with neutral) visual stimuli is associated with enhanced left amygdala rCBF (Dolan et al., 2000). Other neuroimaging studies have reported a positive correlation between amygdala activity during encoding of emotional visual stimuli and subsequent enhanced recall and recognition (Cahill et al., 1996; Hamann et al., 1999). Although our present results are entirely compatible with amygdala involvement in encoding emotional (behaviorally salient) memories, they also suggest, like the data from Dolan et al. (2000), that amygdala function is not confined to this role, but is also involved in memory retrieval processes.

Right anterior orbitofrontal cortex $\mathrm{rCBF}$, by contrast, covaried with recognition memory score for both food and non-food pictures (Fig. 2A,B). This finding accords with results from monkey lesion studies (Bachevalier and Mishkin, 1986; Meunier et al., 1997) and human neuroimaging experiments (Frey and Petrides, 2000) that show orbitofrontal cortex involvement in general visual recognition memory. Entorhinal and perirhinal cortex, also implicated in general visual recognition memory (Meunier et al., 1993), send strong projections to orbitofrontal cortex (Insausti et al., 1987). Right lateral orbitofrontal rCBF, on the other hand exhibited a stimulus-specific interaction with amygdala activity (Fig. 3). It is notable that amygdala has a strong projection to lateral orbitofrontal cortex (area 12o) in the macaque monkey (Carmichael and Price, 1995) and that surgical disconnection of amygdala and orbitofrontal cortex, in a procedure that otherwise leaves these structures intact, disrupts the ability of monkeys to adjust their choice behavior after devaluation of a food reward (Baxter et al., 2000). Moreover, systematic changes in the functional connectivity of amygdala and orbitofrontal cortex have been reported in rat electrophysiological studies of reinforcement learning (Schoenbaum et al., 1998, 2000). Our present findings (Fig. 3) accord with these animal data, therefore, in indicating the importance of connections between amygdala and lateral orbitofrontal cortex in processing biologically salient stimuli (e.g., food).

Hunger-related changes in $\mathrm{rCBF}$ were identified in hypothalamus (Fig. 4). These data accord with a previous human neuroimaging study that reported increased activity in hypothalamus during fasting compared with satiation (Tataranni et al., 1999). Another fMRI study of eating, using temporal clustering analysis, identified a phasic "negative response" in the hypothalamus with a peak 7.7-12.8 min after glucose ingestion (Liu et al., 2000). In accord with the findings of Liu et al. (2000), our neuroimaging data also show sharp decreases in hypothalamic activity occurring $<20$ min after eating (Fig. 4A,B). Additionally, however, progressive increases in hypothalamic $\mathrm{rCBF}$ were evident across scans 5-12 in the early subject group and scans 1-8 in the late group. This temporal pattern suggests that feeding-related hypothalamic responses are not only phasic, but also have a tonic relationship to metabolic variables that influence subjective feelings of hunger.

A similar temporal pattern of hunger-related $\mathrm{rCBF}$ changes was seen in nucleus accumbens in the ventral striatum. The activation in nucleus accumbens was confluent with that observed in hypothalamus (Fig. 4). Experiments in animals have shown that nucleus accumbens is a crucial structure through which natural reinforcers exert their influence on feeding, drinking, and sexual behavior (Wenkstern et al., 1993; Richardson and Gratton, 1996; Taber and Fibiger, 1997). Feeding and hypothalamic stimulation both lead to increased turnover of dopamine in nucleus accumbens (Hernandez and Hoebel, 1988). Our finding of a correlation between nucleus accumbens activity and the motivational state of hunger is consistent, therefore, with these animal data.

Hunger-related $\mathrm{rCBF}$ changes were also observed in insula cortex, a visceral sensory region that may be critical in processing 
A

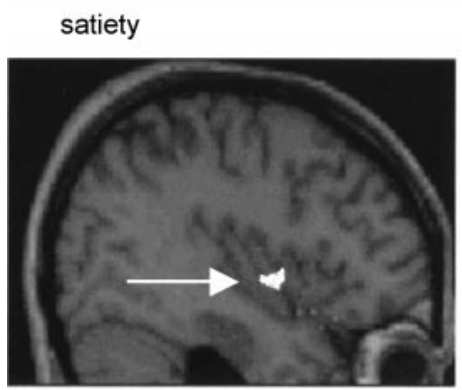

right posterior insula $42,-2,-6$

B

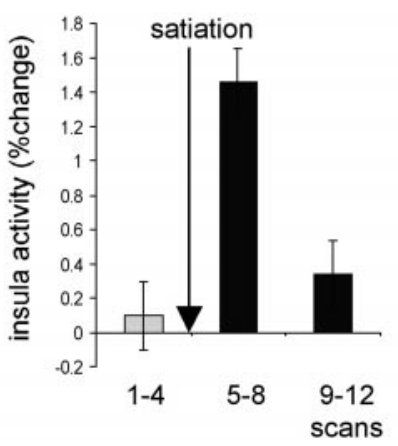

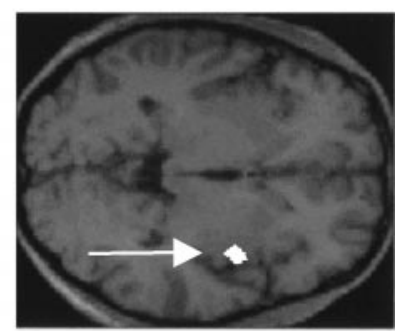
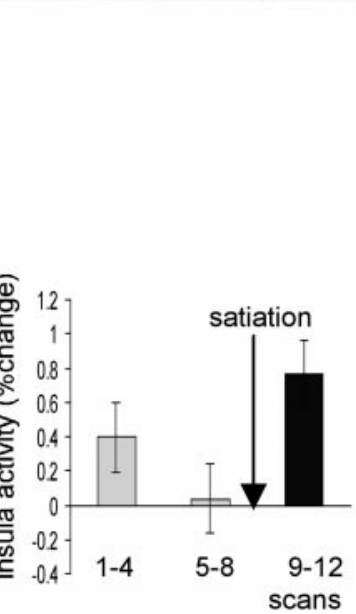

Figure 5. A, An SPM showing an activation in right insula that covaried negatively with subjective ratings of hunger (i.e., covaried positively with satiety). The activation is displayed on sagittal and transverse MRI sections from a representative subject. Right insula is indicated by a white arrow. $B$, Mean insula activity across three scanning blocks (i.e., scans $1-4,5-8$, and 9-12) is shown (in terms of percentage signal change) for early and late satiation groups. Bars represent 2 SEs, and arrows indicate time of satiation.

interoceptive stimuli (Augustine, 1996, Small et al., 1999). It is notable that insula cortex has important anatomical connections with hypothalamus, amygdala, and lateral orbitofrontal cortex (Augustine, 1996). Increased human insula activity during fasting has been reported previously (Tataranni et al., 1999). Whereas our present data are consistent with this earlier study, our results also indicate segregation of feeding-related activity within the insula: pronounced decreases in $\mathrm{rCBF}$ were observed in a right anterior ("hunger") region after eating (Fig. 4C,D), whereas marked increases were seen in a right posterior ("satiety") region (Fig. 5). By contrast, left dorsal insula rCBF was similar to left amygdala, covarying with memory performance for food items. Granular cortex of dorsal insula is known to receive a strong projection from amygdala (Augustine, 1996). The present results provide intriguing evidence, therefore, of both segregation and lateralization of function in the human insula.

The food-specific covariation of right posterior orbitofrontal rCBF with hunger ratings (Fig. 6) accords with both anatomical (Carmichael and Price, 1995, 1996) and electrophysiological (Rolls et al., 1989; Scott et al., 1995; Critchley and Rolls, 1996) data. The extrinsic inputs to posterior orbitofrontal cortex (areas Iam, Iapm, and $\mathrm{G}$ in macaque) are predominantly visceral and gustatory (Carmichael and Price, 1995). However, these posterior regions also receive important intrinsic projections from lateral orbitofrontal area 12 , which in turn receives inputs from both
A

food-hunger interaction
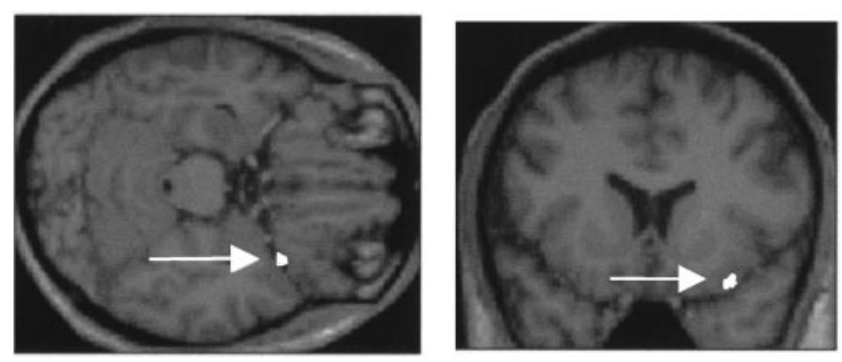

right orbitofrontal cortex

$34,12,-24$
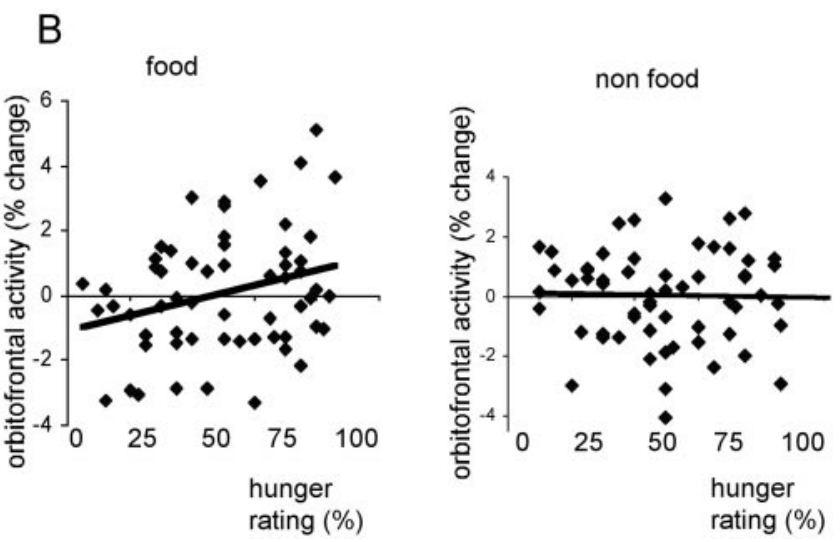

Figure 6. A, An SPM showing an activation in right orbitofrontal cortex that covaried positively with subjective ratings of hunger in the food condition but covaried negatively with hunger in the non-food condition. The activation (indicated by a white arrow) is displayed on sagittal and transverse MRI sections from a representative subject. $B$, Plots of right orbitofrontal activity in food and non-food conditions with respect to hunger ratings. Activity is shown in terms of percentage signal change, and linear regression lines have been fitted to the data.

visual cortex and amygdala (Carmichael and Price, 1996). Moreover, single-unit recordings in monkeys have shown that posterior orbitofrontal responses to food stimuli are modified by hunger and satiety (Rolls et al., 1989; Scott et al., 1995; Critchley and Rolls, 1996). Our observation that rCBF in posterior orbitofrontal cortex is dependent on both hunger ratings and stimulus category (i.e., food vs non-food) is consistent, therefore, with the known functional anatomy of this region (Fig. 6).

The present neuroimaging data comprise several novel findings: hunger-related $\mathrm{rCBF}$ changes in nucleus accumbens, distinct hunger and satiety regions in insula cortex, interactions between perceptual (food-non-food), motivational (hunger), and cognitive (mnemonic) factors reflected by $\mathrm{rCBF}$ changes in distinct regions of orbitofrontal cortex and amygdala, and finally, a stimulus-specific (food-related) psychophysiological interaction between amygdala and orbitofrontal rCBF. These results provide support, therefore, for the proposal that amygdala and orbitofrontal cortex constitute an integrated neural system that is critical for making adaptive responses and guiding decision-making (Bechara et al., 1999; Baxter et al., 2000; Rolls, 2000; Schoenbaum et al., 2000). Our data show, moreover, that other neural structures (e.g., nucleus accumbens) and distinct subregions of other brain areas (e.g., anterior and posterior insula) are also critically involved in mediating physiological and motivational states. Models 
of the neural circuitry underlying adaptive behavior will need to reflect the complexity, therefore, of both extrinsic (e.g., amygdala-orbitofrontal, amygdala-insula, amygdala-accumbens), and intrinsic (e.g., intra-orbitofrontal, intra-insula, intraamygdala) functional connections.

\section{REFERENCES}

Adolphs R, Tranel D, Denburg N (2000) Impaired emotional declarative memory following unilateral amygdala damage. Learn Mem 7:180-186.

Augustine AR (1996) Circuitry and functional aspects of the insular lobe in primates including humans. Brain Res Rev 22:229-244.

Bachevalier J, Mishkin M (1986) Visual recognition impairment follows ventromedial but not dorsolateral prefrontal lesions in monkeys. Behav Brain Res 20:249-261.

Baxter MG, Parker A, Lindner CAA, Izquierdo AD, Murray EA (2000) Control of response selection by reinforcer value requires interaction of amygdala and orbitofrontal cortex. J Neurosci 20:4311-4319.

Bechara A, Damasio H, Damasio AR, Lee GP (1999) Different contributions of the human amygdala and ventromedial prefrontal cortex to decision-making. J Neurosci 19:5473-5481.

Cahill L, McGaugh JL (1998) Mechanisms of emotional arousal and lasting declarative memory. Trends Neurosci 21:294-299.

Cahill L, Haier RJ, Fallon J, Alkire MT, Tang C, Keator D, Wu J, McGaugh JL (1996) Amygdala activity at encoding correlated with long-term, free recall of emotional information. Proc Natl Acad Sci USA 93:8016-8021.

Canli T, Zhao Z, Brewer J, Gabrieli JDE, Cahill L (2000) Event-related activation in the human amygdala associates with later memory for individual emotional experience. J Neurosci 20:RC99:1-5.

Carmichael ST, Price JL (1995) Limbic connections of the orbital and medial prefrontal cortex in the macaque monkey. J Comp Neurol 346:366-402.

Carmichael ST, Price JL (1996) Connectional networks within the orbital and medial prefrontal cortex of macaque monkeys. J Comp Neurol 371:179-207.

Critchley HD, Rolls ET (1996) Hunger and satiety modify the responses of olfactory and visual neurons in the primate orbitofrontal cortex. J Neurophysiol 75:1673-1686.

Dolan RJ, Lane R, Chua P, Fletcher P (2000) Dissociable temporal lobe activations during emotional episodic retrieval. NeuroImage 11:203-209.

Erwin RJ, Ferguson ED (1979) The effect of food and water deprivation and satiation on recognition. Am J Psychol 92:611-626.

Ferguson ED (1983) The effect of motivation and word characteristics on recognition. Am J Psychol 96:253-266.

Frey S, Petrides M (2000) Orbitofrontal cortex: a key prefrontal region for encoding information. Proc Natl Acad Sci USA 97:8723-8727.

Friston KJ, Holmes AP, Worsley KJ, Poline J-P, Frith CD, Frackowiak RSJ (1995) Statistical parametric maps in functional imaging: a general linear approach. Hum Brain Mapp 2:189-210.

Friston KJ, Buechel C, Fink G, Morris JS, Rolls ET, Dolan RJ (1997) Psychophysiological and modulatory interactions in neuroimaging. NeuroImage 6:218-229.

Hamann SB, Ely TD, Grafton SC, Kilts CD (1999) Amygdala activity related to enhanced memory for pleasant and aversive stimuli. Nat Neurosci 2:289-293

Hernandez L, Hoebel BG (1988) Feeding and hypothalamic stimulation increase dopamine turnover in the accumbens. Physiol Behav 44:599-606.

Insausti R, Amaral DG, Cowan WM (1987) The entorhinal cortex of the monkey: II. Cortical afferents J Comp Neurol 264:356-395.

LeDoux JE (2000) Emotion circuits in the brain. Annu Rev Neurosci 23:155-184.

Liu Y, Gao J-H, Liu H-L, Fox PT (2000) The temporal response of the brain after eating revealed by functional MRI. Nature 405:1058-1062.

Meunier M, Bachevalier J, Mishkin M, Murray EA (1993) Effects on visual recognition of combined and separate ablations of the entorhinal and perirhinal cortex in rhesus monkeys. J Neurosci 13:5418-5432.

Meunier M, Bachevalier J, Mishkin M (1997) Effects of orbital frontal and anterior cingulate lesions on object and spatial memory in rhesus monkeys. Neuropsychologia 35:999-1015.

Nishijo H, Ono T, Nishino H (1988) Single neuron responses in amygdala of alert monkey during complex sensory stimulation with affective significance. J Neurosci 8:3570-3583.

Richardson NR, Gratton A (1996) Behavior-relevant changes in nucleus accumbens dopamine transmission elicited by food reinforcement: an electrochemical study in rat. J Neurosci 16:8160-8169.

Rolls ET (2000) The orbitofrontal cortex and reward. Cereb Cortex 10:284-294.

Rolls ET, Rolls BJ, Rowe EA (1982) Sensory-specific and motivationspecific satiety for the sight and taste of food and water in man. Physiol Behav 30:185-192.

Rolls ET, Sienkiewicz ZJ, Yaxley S (1989) Hunger modulates the responses to gustatory stimuli of single neurons in the caudolateral orbitofrontal cortex of the macaque monkey. Eur J Neurosci 1:53-60.

Rolls ET, Yaxley S, Sienkiewicz ZJ (1990) Gustatory responses of single neurons in the orbitofrontal cortex of the macaque monkey. J Neurophysiol 64:1055-1066.

Sanghera MK, Rolls ET, Roper-Hall A (1979) Visual responses of neurons in the dorsolateral amygdala of the alert monkey. Exp Neurol 63:610-626.

Schoenbaum G, Chiba AA, Gallagher M (1998) Orbitofrontal cortex and basolateral amygdala encode expected outcomes during learning. Nat Neurosci 1:155-159.

Schoenbaum G, Chiba AA, Gallagher M (2000) Changes in functional connectivity in orbitofrontal cortex and basolateral amygdala during learning and reversal training. J Neurosci 20:5179-5189.

Scott TR, Yan J, Rolls ET (1995) Brain mechanisms of satiety and taste in macaques. Neurobiology 3:281-292.

Small DA, Zald DH, Jones-Gotman M, Zatorre RJ, Pardo JV, Frey S, Petrides M (1999) Human cortical gustatory areas: a review of functional neuroimaging data. NeuroReport 10:7-14.

Taber MT, Fibiger HC (1997) Feeding-evoked dopamine release in the nucleus accumbens: regulation by glutaminergic mechanisms. Neuroscience 76:1105-1112.

Tataranni PA, Gautier J-F, Chen K, Ueker A, Bandy D, Salbe AD, Pratley RE, Lawson M, Reiman EM, Ravussin E (1999) Neuroanatomical correlates of hunger and satiation in humans using positron emission tomography. Proc Natl Acad Sci USA 96:4569-4574.

Wenkstern D, Pfaus JG, Fibiger HC (1993) Dopamine transmission increases in the nucleus accumbens of male rats during their first exposure to sexually receptive female rats. Brain Res 618:41-46.

Worsley KJ, Marrett P, Neelin AC, Friston KJ, Evans AC (1996) A unified statistical approach for determining significant signals in images of cerebral activation. Hum Brain Mapp 4:58-73. 